\title{
Pi 2 waves simultaneously observed by Cluster and CPMN ground-based magnetometers near the plasmapause
}

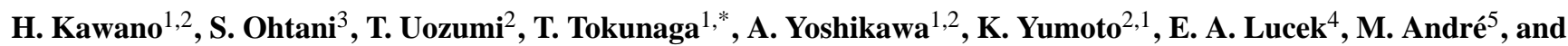 \\ the CPMN group ${ }^{6}$ \\ ${ }^{1}$ Department of Earth and Planetary Sciences, Kyushu University, Fukuoka, Japan \\ ${ }^{2}$ Space Environment Research Center, Kyushu University, Fukuoka, Japan \\ ${ }^{3}$ Johns Hopkins University, Applied Physics Laboratory, Laurel, USA \\ ${ }^{4}$ Space and Atmospheric Physics Group, Blackett Laboratory, Imperial College, London, UK \\ ${ }^{5}$ Swedish Institute of Space Physics, Uppsala, Sweden \\ ${ }^{6}$ http://denji102.geo.kyushu-u.ac.jp/denji/obs/cpmn/cpmn_obs_e.html \\ * now at: Meiji Institute for Advanced Study of Mathematical Sciences, Kawasaki, Japan
}

Received: 12 January 2011 - Revised: 13 June 2011 - Accepted: 1 August 2011 - Published: 28 September 2011

\begin{abstract}
We have analyzed an event on 14 February 2003 in which Cluster satellites and the CPMN ground magnetometer chain made simultaneous observations of a Pi 2 pulsation along the same meridian. Three of the four Cluster satellites were located outside the plasmasphere, while the other one was located within the plasmasphere. By combining the multipoint observations in space and the multipoint observations on the ground, we have obtained a detailed $L$-profile of the Pi 2 signatures, which has not been done in the past. In addition, we have used a method called Independent Component Analysis (ICA) to separate out other superposed waves with similar spectral components. The result shows that the wave phase of the Pi 2 was the same up to $L \sim 3.9$ (corresponding to the plasmasphere), became earlier up to $L \sim 4.1$ (corresponding to the plasmapause boundary layer), and showed a delaying tendency up to $L \sim 5.9$ (corresponding to the plasmatrough). This systematic phase pattern, obtained for the first time by a combination of a ground magnetometer chain and multisatellites along a magnetic meridian with the aid of ICA, supports the interpretation that a Pi 2 signal propagated from a farther source and reached the plasmasphere.
\end{abstract}

Keywords. Magnetospheric physics (Magnetotail boundary layers; MHD waves and instabilities; Plasmasphere)

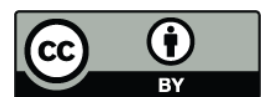

Correspondence to: H. Kawano (hkawano@geo.kyushu-u.ac.jp)

\section{Introduction}

The Pi 2 pulsation is the transient wave phenomenon observed in space and on the ground at the start time of a magnetospheric substorm (e.g. Olson, 1999) or a pseudo-breakup (Hsu and McPherron, 2007). Pi 2 has its period in the range of 40-150s, typically has a damping-type waveform, and typically continues for a few minutes to $\sim 10 \mathrm{~min}$.

There exist many publications as regards the source region and propagation of Pi 2. Among them, Uozumi et al. (2004, 2007) statistically analyzed Pi 2 's observed at high-latitude ground magnetometers belonging to the Circum-pan Pacific Magnetometer Network (CPMN) (Yumoto and the 210MM Magnetic Observation Group, 1996; Yumoto and the CPMN Group, 2001) to estimate the source region of Pi 2 to be located in space at $9 \operatorname{Re}$ (from the geocenter) and 22.5 MLT; they assumed that Pi 2 propagated as fast-mode and Alfven waves from the source region down to the ground high latitudes, and made model calculations that best-matched the observed time lags among the ground stations they used. Chi et al. (2009) analyzed four Pi 2's observed at a meridian chain of ground magnetometers located at $300-330^{\circ}$ magnetic longitudes in the north-American continent. For each Pi 2, they fit their Pi 2-propagation model to estimate the onset position; as a result, they obtained $X \sim-20 R_{\mathrm{E}}$.

On the other hand at low latitudes on the ground, it is well known that ground magnetometers observe Pi 2 waves simultaneously and in-phase; this led researchers to think that the inner magnetosphere oscillates as a whole, and they called this type of oscillation the cavity mode (e.g. Yeomen and Orr, 1989; Sutcliffe and Yumoto, 1991).

Published by Copernicus Publications on behalf of the European Geosciences Union. 
There remain arguments about the outer boundary of the cavity mode oscillation, but a popular idea is that the plasmapause is the outer boundary: Takahashi et al. (2003) performed a detailed statistical analysis of the magnetic and electric field data from the CRRES satellite, and concluded that the node of the cavity mode is located near the plasmapause.

The above-cited source region of Pi 2 is located outside the plasmasphere; thus, a question has been how the cavity mode is excited by the outside source. A scenario has been that an impulse from the source region propagates inward and hits the magnetospheric cavity (e.g. Yeoman and Orr, 1989). However, the actual process of this hitting was not observed well in space in the 20th century, because a single satellite was not good enough to separate out the temporal effects from the spatial effects near the plasmapause. The 2000 launch of Cluster, consisting of four identical satellites (Escoubet et al., 2001), provided for the first time in history chances for simultaneously observing the region near the plasmapause by multiple satellites.

Collier et al. (2006) were the first to analyze a case in which Cluster satellites were located near the plasmapause during a Pi 2 event; three of the four satellites were located within the plasmasphere (CL1, CL2, and CL4 at $L=4.7,4.5$, and 4.6) while the fourth (CL3 at $L=6.6$ ) was located at or just outside the plasmapause. As a result of the analysis they found in the data of the three satellites in the plasmasphere evidence for the standing wave in both the radial and fieldaligned directions, strongly indicative of the cavity mode. On the other hand, the magnetic field perturbation observed at CL3 preceded the activity at the other three satellites (located within the plasmasphere), which may be attributed to the time interval required for inward radial propagation of the Pi 2 signal; though, they also stated that the perturbation at CL3 was highly irregular, quite distinct from those at the other three satellites. They stated that one cannot exclude the possibility that the Pi 2 event at CL3 was masked by other waves with similar spectral content.

In this paper we have also found a Pi 2 event for which Cluster satellites were located near the plasmapause; we also analyze data from the four Cluster satellites. In addition, as a new feature of this paper, we also use simultaneously obtained data at a ground magnetometer chain: the Cluster magnetic footprints were located close (in longitude) to the meridian of the ground magnetometers at the time of the Pi 2 event, and thus, by field-aligned mapping the Cluster data to the ground and analysing them along with the data from the ground magnetometer chain, we will be able to understand the $L$ dependence of the event in a systematic manner. Also, we will use a method called Independent Component Analysis (ICA) to separate out other waves with similar spectral content.

\section{Data}

\subsection{Cluster spacecraft}

Cluster consists of four identical satellites, which were launched in 2000 (Escoubet et al., 2001). The four satellites were kept close to each other in space. The orbital planes of the satellites were also close to each other and the normal vectors of the orbital planes were fairly perpendicular to the earth's axis. At the launch, the geocentric distances of the apogees (perigees) of the Cluster spacecraft were $\sim 20(\sim 4) R_{\mathrm{E}}$; these small perigees enabled Cluster to sometimes enter the plasmasphere, and the event of this paper is such an example.

In this paper we show the magnetic field data measured by the FGM instrument (Balogh et al., 2001) and the spacecraft potential data measured by the EFW instrument (Gustafsson et al., 1997), both on board the Cluster spacecraft. The data were retrieved from the Cluster Active Archive (http://caa. estec.esa.int/caa/) with time resolution of $0.2 \mathrm{~s}(4 \mathrm{~s})$ for the magnetic field data (spacecraft potential data).

For the magnetic field data analysis of Pi 2 waves, we will use mean-field-aligned (MFA) coordinates (e.g. Takahashi et al., 2001; Collier et al., 2006). In this system, $\boldsymbol{e}_{\mathrm{Z}}$ is in the direction of the mean magnetic field, which is defined by $150-\mathrm{s}$ moving averages of the 0.2-s magnetic field data; $\boldsymbol{e}_{\mathrm{y}}$, is parallel to $\boldsymbol{e}_{\mathrm{Z}} \times \boldsymbol{r}$, where $\boldsymbol{r}$ is the vector from the geocenter to the satellite; and $\boldsymbol{e}_{\mathrm{x}}$ is set so as to complete the $\left(\boldsymbol{e}_{\mathrm{x}}, \boldsymbol{e}_{\mathrm{y}}, \boldsymbol{e}_{\mathrm{z}}\right)$ triad. Finally, the 0.2-s data in MFA coordinates are averaged over $3 \mathrm{sec}$ and will be used for the Pi 2 analysis of this paper.

\subsection{CPMN ground magnetometers}

For comparisons with the above-stated Cluster data, we use simultaneously observed ground magnetometer data from the Circum-pan Pacific Magnetometer Network (CPMN) (Yumoto and the 210MM Magnetic Observation Group, 1996; Yumoto and the CPMN Group, 2001), mainly run by the Space Environment Research Center (SERC), Kyushu University, Japan. We use 3-s $H$-component data from CPMN stations along the $210^{\circ}$ magnetic meridian (MM). The locations of the stations are listed in Table 1 . For each ground station, the table shows its geographic (GEO) coordinates, AACGM (Baker and Wing, 1989) coordinates, and $L$-value calculated by using the equation $L=1 /(\cos \lambda)^{2}$ where $\lambda$ refers to the AACGM latitude. Table 1 also shows the location information of the four Cluster satellites.

\section{Data analysis}

Figure 1 shows the orbits of the Cluster satellites for the interval 13:00-15:00 UT on 14 February 2003, in GSE coordinates. As stated below, the Pi 2 of this paper took place around 14:02:02 UT; at this time, the Cluster satellites were located on the nightside and were flying near their perigees. 
Table 1. Geographic and geomagnetic coordinates of the CPMN ground stations and the Cluster satellites used in this paper. Geographic coordinates of the Cluster satellites are those of the footprints of the satellites at 14:02:02 UT on 14 February 2003, calculated by using the Tsyganenko 96 model. Geomagnetic coordinates of the ground stations and the Cluster footprints are calculated by using AACGM. The $L$-value is calculated by using the equation $L=1 /(\cos \lambda)^{2}$ where $\lambda$ refers to the AACGM latitude. In-situ locations of the Cluster satellites, in geocentric distance $(r)$ and geomagnetic coordinates (latitude and longitude), are also shown in the rightmost three columns. See the text for more details.

\begin{tabular}{lcc|cc|c|c|c|cc}
\hline & \multicolumn{5}{c|}{ CPMN or Cluster footprints } & \multicolumn{3}{|c|}{ Cluster in-situ } \\
\cline { 2 - 7 } & \multicolumn{2}{c}{ Geographic } & \multicolumn{2}{c|}{ AACGM } & $L$ & $r$ & \multicolumn{2}{|c}{ geomagnetic } \\
& lat & long & lat & long & & & lat & long \\
\hline TIK & 71.59 & 128.78 & 65.76 & 196.87 & 5.93 & & & \\
CL1 & 69.82 & 178.74 & 65.46 & 234.52 & 5.80 & 4.82 & 29.70 & 235.65 \\
CHD & 70.62 & 147.89 & 64.79 & 212.19 & 5.51 & & & \\
CL2 & 67.72 & 178.26 & 63.18 & 235.75 & 4.91 & 4.46 & 24.57 & 236.77 \\
CL4 & 65.15 & 178.17 & 60.41 & 237.27 & 4.10 & 4.07 & 16.90 & 238.18 \\
ZYK & 65.75 & 150.78 & 59.74 & 216.91 & 3.94 & & & \\
CL3 & 64.28 & 180.76 & 59.72 & 239.59 & 3.93 & 4.28 & -2.76 & 240.93 \\
MGD & 59.97 & 150.86 & 53.60 & 218.94 & 2.84 & & & \\
PTK & 52.94 & 158.25 & 46.18 & 226.21 & 2.09 & & & \\
GAM & 13.58 & 144.87 & 5.82 & 215.61 & 1.01 & & & \\
BSV & -25.54 & 139.21 & -36.01 & 213.09 & 1.53 & & & \\
ADL & -34.67 & 138.65 & -46.13 & 213.83 & 2.08 & & & \\
\hline
\end{tabular}
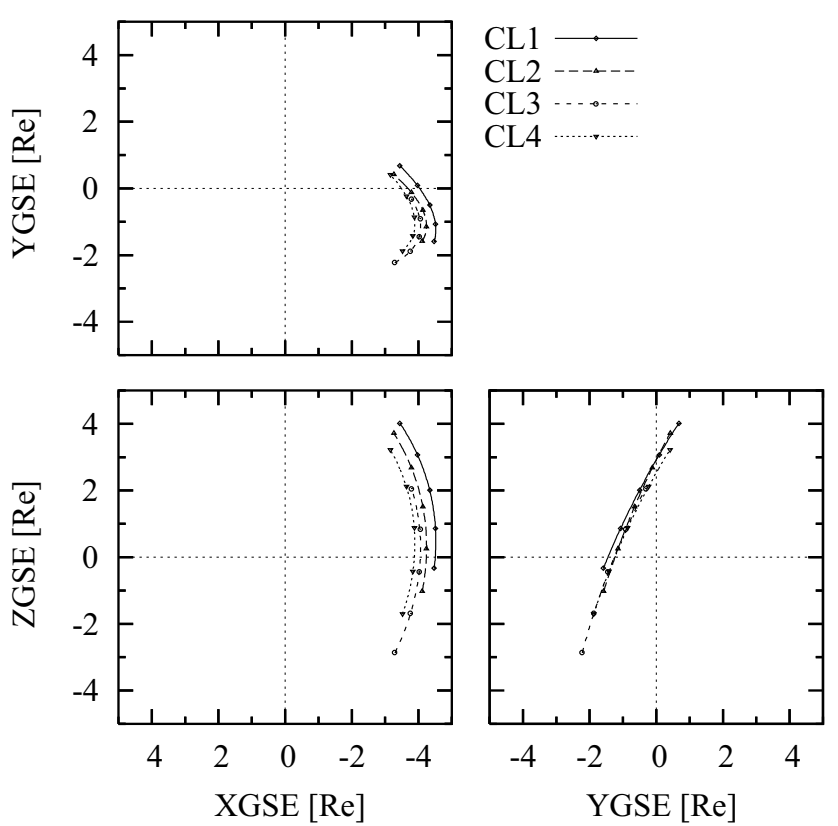

Fig. 1. Shows the orbital segments of the Cluster satellites for the interval 13:00-15:00 UT on 14 February 2003, in GSE coordinates. The orbital information was taken from the SSCweb (http://sscweb.gsfc.nasa.gov/). All the satellites moved upward in the bottom two panels. Different symbols are plotted for different satellites, at 13:00, 13:30, 14:00, 14:30, and 15:00 UT.
Rightmost three columns of Table 1 list the locations of the Cluster satellites in space at 14:02:02 UT, in terms of the geocentric distance, geomagnetic (MAG) latitude, and MAG longitude.

For each Cluster satellite, Table 1 also shows the location of its footprint, field-aligned traced by using the Tsyganenko 96 (T96) model (Tsyganenko, 1996). The $L$ value of the Cluster footprint, shown in the table, is calculated by using the equation $L=1 /(\cos \lambda)^{2}$ where $\lambda$ refers to the AACGM latitude of the footprint. The T96 model parameters are set as follows: $\mathrm{DP}=3.4 \mathrm{nPa}, \mathrm{Dst}=-3 \mathrm{nT}$, $\mathrm{IMF} B_{\mathrm{y}}=8.7 \mathrm{nT}$, and IMF $B_{\mathrm{z}}=-0.7 \mathrm{nT}$; these values were measured by the ACE spacecraft (http://www.srl.caltech.edu/ ACE/ASC/index.html) except that Dst originally came from NSSDC Kyoto (http://wdc.kugi.kyoto-u.ac.jp/). In more detail, since the magnetosphere responds relatively gradually to changes in these parameters, we have used OMNI hourly averages of these quantities available on CDAWeb (http://cdaweb.gsfc.nasa.gov/); we have averaged the data corresponding to two intervals 13:00-14:00 UT and 14:0015:00 UT (in OMNI data, the propagation lag from ACE to the magnetosphere is already taken into account). We note in Table 1 that the footprints of the Cluster satellites are close to the CPMN stations in longitude (see AACGM long).

Figure 2 shows the negative of the spacecraft potential ( $\mathrm{SP}^{-}$below) measured by the EFW experiment on board the Cluster spacecraft. The $\mathrm{SP}^{-}$positively correlates with the plasma density around the spacecraft (lower $\mathrm{SP}^{-}$corresponds to lower density) (Pedersen et al., 2008). However, when the Active Spacecraft Potential Control (ASPOC) ion emitter (Torkar et al., 2001) on board Cluster is activated, the 


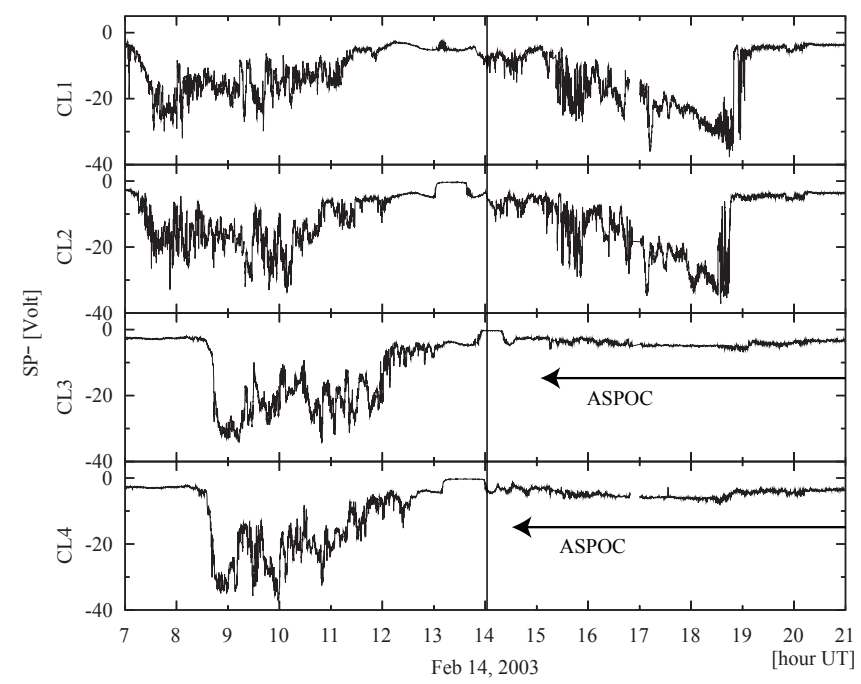

Fig. 2. Shows the negative of the spacecraft potential $\left(\mathrm{SP}^{-}\right)$measured by the EFW experiment on board the Cluster spacecraft (from top, Cluster CL1, CL2, CL3, and CL4). The vertical line is drawn at the onset time of the Pi 2 analyzed in this paper (14:02:02 UT). See the text for more details.

$\mathrm{SP}^{-}$becomes flat near zero. The ASPOC intervals for CL3 and CL4 are shown by superposed horizontal lines in Fig. 2 (ASPOC of CL1 and CL2 was not activated during the interval of the figure).

Figure 2 shows that, for all the four satellites, the $\mathrm{SP}^{-}$'s were large near the beginning and the end of the time interval of the figure; for these intervals the satellites were located within the magnetosheath-solar wind. Next to these magnetosheath-solar wind intervals, the satellites were located within the tail lobe-polar cap, thus the $\mathrm{SP}^{-}$'s were much smaller.

Near the centre of Fig. 2, the $\mathrm{SP}^{-}$'s were large for all the satellites; for these intervals the satellites were located within the plasmasheet-plasmasphere. In particular, the rectangular bumps ( 13:10 UT for CL1, 13:00-13:40 UT for CL2, 13:50-14:25 UT for CL3, and 13:10-14:00 UT for CL4) likely correspond to the entries of the satellites into the plasmasphere when they were near their perigees; this interpretation is consistent with the region information we can obtain from the quick-look energy-time ion spectrograms from the CIS instrument onboard Cluster (Rème et al., 2001) (not shown here) (The quicklooks are found at http://cluster.cesr. fr:8000/public/spectro/index.php?vue=SCI).

The vertical line in Fig. 2 marks the Pi 2 onset time (14:02:02 UT; discussed below by using Fig. 3). At this Pi 2 onset time, CL1, CL2, and CL4 were located outside the plasmasphere while CL3 was located inside the plasmasphere, as shown by the above-mentioned bumps.

Figure 3 is an overview plot of the ground Pi 2 signatures; shown are the $H$-component data from the CPMN stations listed in Table 1, bandpass filtered with the Pi 2 period range

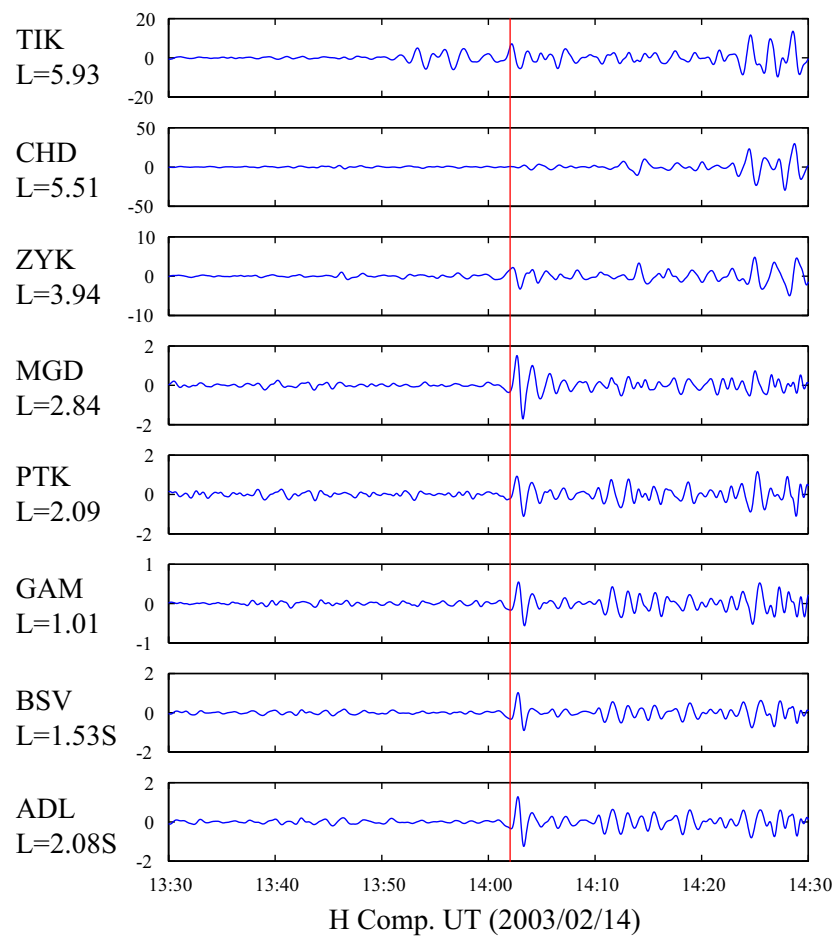

Fig. 3. Overview plot of the ground Pi 2 signatures; shown are the $H$-component data from the CPMN stations listed in Table 1, bandpass filtered with the Pi 2 period range (40-150s). The Pi 2 onset time, 14:02:02 UT, determined by visual inspection of the GAM data, is shown by the vertical line. See the text for more details.

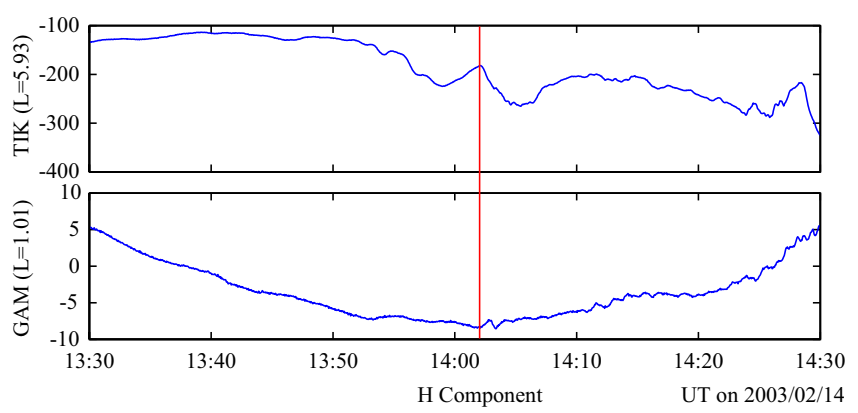

Fig. 4. Shows the raw data plot of the $H$-component magnetometer data from the CPMN stations TIK and GAM. See Table 1 for their locations. The vertical line is drawn at the onset time of the Pi 2 analyzed in this paper (14:02:02 UT). See the text for more details.

(40-150 s). The Pi 2 onset time, 14:02:02 UT, determined by visual inspection of the GAM data, is shown by the vertical line. The Pi 2 signal is clearly identified at ZYK through ADL.

Figure 4 shows the raw data plot of the $H$-component magnetometer data from the CPMN stations TIK and GAM. The vertical line marks the Pi 2 onset time (14:02:02 UT); at this time, a small but clear negative bay started at TIK (located at high latitudes; see Table 1), and a small but 

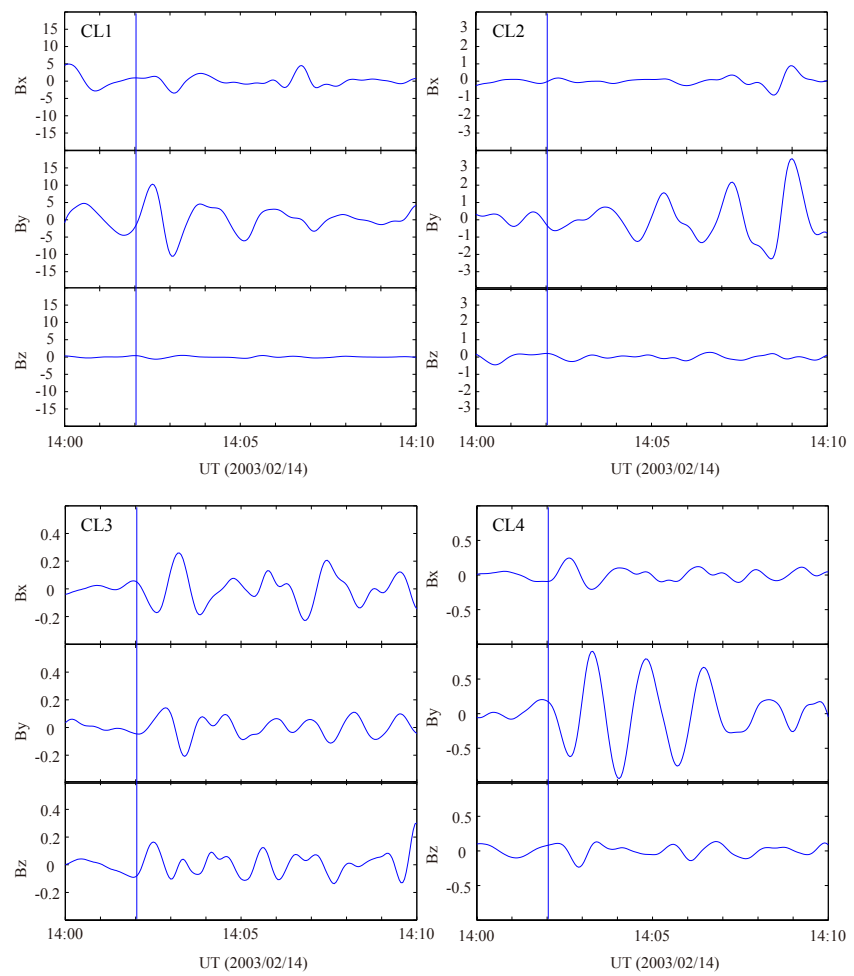

Fig. 5. Shows the bandpass-filtered (40-150 s) magnetic field data of the Pi 2 of this paper observed by CL1 (top left), CL2 (top right), CL3 (bottom left), and CL4 (bottom right). The magnetic field vector is expressed in the mean-field-aligned (MFA) coordinates. The Pi 2 onset time (14:02:02 UT) is shown by the vertical lines. See the text for more details.

identifiable positive bay started at GAM (located at low latitudes). We also note that LANL geosynchronous satellite 1994-084 (which was located at 23:36 LT at 14:02 UT) observed a signature of a low-energy particle injection (not shown here) (the 1994-084 data are available at CDAWeb: http://cdaweb.gsfc.nasa.gov/). Thus, we can say that this Pi 2 took place at the start time of a substorm.

Figure 5 shows the bandpass-filtered (40-150 s) magnetic field data from the four Cluster satellites for the 10-min interval including the Pi 2 introduced above. The magnetic field vector is expressed in the MFA coordinates. Note that the vertical axis range is different from satellite to satellite, although it is the same for the three subpanels (showing $B_{\mathrm{x}}$, $B_{\mathrm{y}}$, and $B_{\mathrm{z}}$ ) in each panel. From this figure one can see that $B_{\mathrm{y}}$ is generally larger than $B_{\mathrm{x}}$ and $B_{\mathrm{z}}$ in amplitude, and that the waveforms looks different from satellite to satellite and from component to component.

To study the relation between such Cluster signatures and the CPMN ground signatures, we have first performed the correlation analysis, as follows. First, by visual inspection, we have selected the interval during which the Pi 2 in question was significant at all the four Cluster satellites. The resultant interval is 14:01:30-14:09:37 UT (Cluster data plots

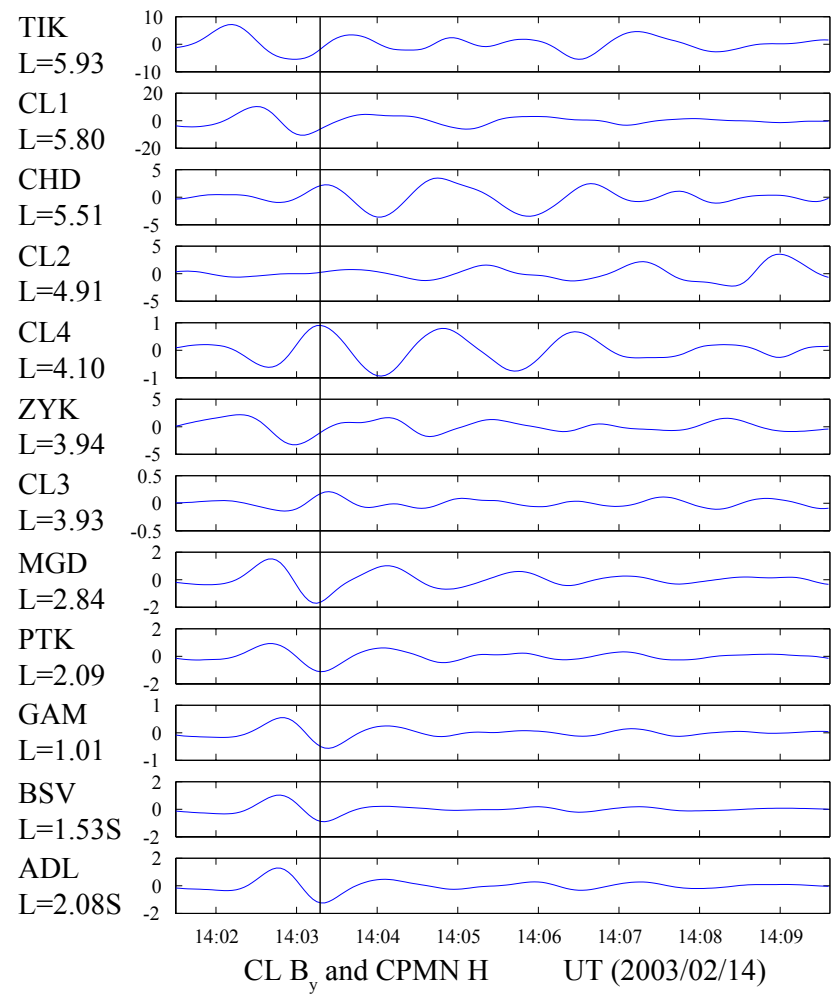

Fig. 6. Shows, in the order of $L$, the bandpass-filtered data of CPMN ground $H$ and Cluster $B_{\mathrm{y}}$ (eastward component in the MFA coordinates). The period range of the bandpass filter is 40-150 s. The vertical line is meant as a guide, and is plotted so as to run through a minimum at MGD-ADL. See the text for more details.

for this interval will be shown below); the number of 3-sresolution Cluster data in this interval is 163 .

We have then calculated the coherence between GAM $H$ and CL $B_{\mathrm{x}}$, the coherence between GAM $H$ and CL $B_{\mathrm{y}}$, and the coherence between GAM $H$ and CL $B_{\mathrm{z}}$ for each of the four Cluster satellites, for the interval 14:01:30 14:09:37 UT; here we are using the data from GAM, located near the magnetic equator, as the reference ground data. The coherence is calculated by using FFT, with a data window of 128 datapoints (i.e. 384 s) slided by every eight datapoints. From thus obtained results, we use the coherence at a period of $96 \mathrm{~s}$, at which the GAM $H$ data during the interval 14:01:30-14:09:37 UT had the maximum power.

Thus obtained coherence was larger than 0.6 for CL4 $B_{\mathrm{y}}$, CL3 $B_{\mathrm{y}}$, CL3 $B_{\mathrm{x}}$, CL1 $B_{\mathrm{x}}$, CL1 $B_{\mathrm{y}}$, and CL2 $B_{\mathrm{y}}$; that is, these six components are regarded as significantly correlated with GAM $H$. (This threshold 0.6 is often used in Pi 2 studies; see, e.g. Takahashi et al., 1995.) It is to be noted that, at all the four Cluster satellites, the $B_{\mathrm{y}}$ component is significantly correlated with the ground $H$ component. On the other hand, only two satellites show significant correlation in $B_{\mathrm{X}}$, and no satellite shows significant correlation in $B_{\mathrm{z}}$. In this paper we want to study the correlation between the ground stations and 
the four Cluster satellites, and for this purpose $B_{\mathrm{x}}$ and $B_{\mathrm{Z}}$ are insufficient. Thus, in this paper we concentrate on $B_{\mathrm{y}}$.

Figure 6 shows the bandpass-filtered data of CPMN ground $H$ and Cluster $B_{\mathrm{y}}$, in the same time frame as that used for the above-stated coherence analysis (i.e. 14:01:30 14:09:37 UT), in the order of $L$. The vertical line is meant as a guide, and is plotted so as to run through a minimum at MGD-ADL. It is to be noted that CL3 $B_{\mathrm{y}}$ is reversed in its sign; it is made so because only CL3 was in the Southern Hemisphere $\left(Z_{\mathrm{MAG}}=-0.2 \mathrm{Re}\right.$ on 14:02 UT) while the other three Cluster satellites were in the Northern Hemisphere $\left(Z_{\mathrm{MAG}}=2.4,1.9\right.$, and 1.2 for CL1, CL2, and CL4).

In Fig. 6, the low-latitude stations (MGD-ADL) show coherent perturbations: this feature has frequently been reported in publication and has often been regarded as a manifestation of the cavity mode (e.g. Yumoto and the CPMN group, 2001; Takahashi et al., 2003). It is to be noted that the plasmapause was located between CL4 and CL3 (see Fig. 2); thus, MGD-ADL are all likely to have been within the plasmasphere. On the other hand, the waveforms at higher latitudes look very different from those at low latitudes, which complicates further analysis. Here we expect that the higherlatitude data include signals similar to those at low latitudes, but superposed signals complicate the higher-latitude data; then, to extract the common part, we use the method called the Independent Component Analysis (ICA), which is explained below.

When signals having different characteristics are mixed, each signal is naturally regarded as being independent from the others. Thus, ICA uses a statistical-mathematics approach to separate out different signals included in a timeseries by maximizing the independence of each signal. ICA has been proven to be useful, for example, for cases in which one wants to separate out several speakers' voices when they speak at the same time (examples are found in the textbook by Hyvärinen et al., 2001). As the computer algorithm of ICA, in this paper we use FastICA (Hyvärinen, 1999) the software of which is found at http://www.cis.hut.fi/projects/ ica/fastica/. FastICA uses the algorithm called "fixed-point algorithm" to maximize the independence of signals to be separated out. We also note that Tokunaga et al. (2007) applied FastICA to ground magnetometer data of a Pi 2 event for the first time in space physics.

Figure 7 shows the result of applying ICA to the bandpassfiltered $H$-component data from the eight CPMN stations (as shown in Fig. 6) and bandpass-filtered $B_{\mathrm{x}}, B_{\mathrm{y}}$ and $B_{\mathrm{z}}$ from the four Cluster satellites (i.e. applying ICA to $8+3 \times 4=20$ timeseries) in the same time frame as that used for the abovestated coherence analysis (i.e. 14:01:30-14:09:37 UT). Here we are using two independent components (IC's) that well reproduce the waveforms of low- to mid-latitude ground $H$ (ZYK-ADL); each of the two IC's (not shown) has a waveform similar to the observed Pi 2, and the phases of the two IC's are different from each other, so that the combination of the two IC's can express the time lag between different

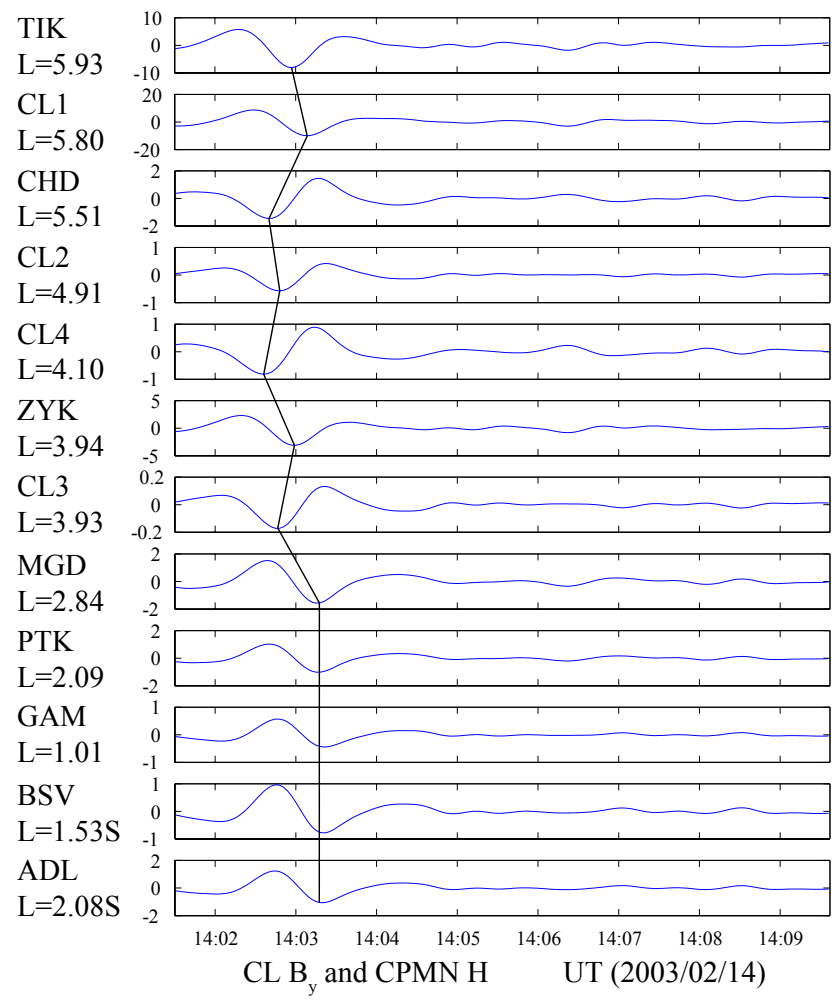

Fig. 7. Shows the result of applying ICA to the bandpass-filtered $H$-component data from the eight CPMN stations (as shown in Fig. 6) and bandpass-filtered $B_{\mathrm{X}}, B_{\mathrm{y}}$ and $B_{\mathrm{Z}}$ from the four Cluster satellites. Superposed line segments pass through a minimum in each timeseries data. See the text for more details.

datasets (such as that between ZYK and MGD, which is clear in Fig. 6). It is to be noted that Fig. 7 shows only $B_{\mathrm{y}}$ from the four Cluster satellites, even though ICA was also applied to $B_{\mathrm{x}}$ and $B_{\mathrm{Z}}$ at once (in order to best extract the common feature in the available data with ICA). Superposed line segments pass through a minimum in each timeseries data.

If we try to understand the observed phase pattern in terms of the Pi 2 propagation effect, we have to take into account the propagation time from Cluster to the ground along the magnetic field line. To calculate it, we use the procedure of Uozumi et al. (2000, 2007): we assume a cold plasma consisting of hydrogen ions and electrons, use the Tsyganenko 96 model for the magnetic field, use the model of Carpenter and Anderson (1992) for the equatorial plasma density, and use the power-law model for the field-aligned distribution of the plasma density $n$, i.e. $n(r)=n_{\mathrm{eq}}\left(r / r_{\mathrm{eq}}\right)^{-p}$, where $r$ is the geocentric distance of any point on a field line, $r_{\mathrm{eq}}$ is $r$ on the equatorial plane, $n_{\mathrm{eq}}$ is $n$ there, and $p$ is set to 3 (4) inside (outside) the plasmapause (Takahashi and Anderson, 1992). By using these models we have calculated the Alfven velocity $\left(V_{\mathrm{A}}\right)$ distribution along the field line running through Cluster (CL1, CL2, CL3, or CL4), and then calculated the Alfven wave travel time from Cluster to 


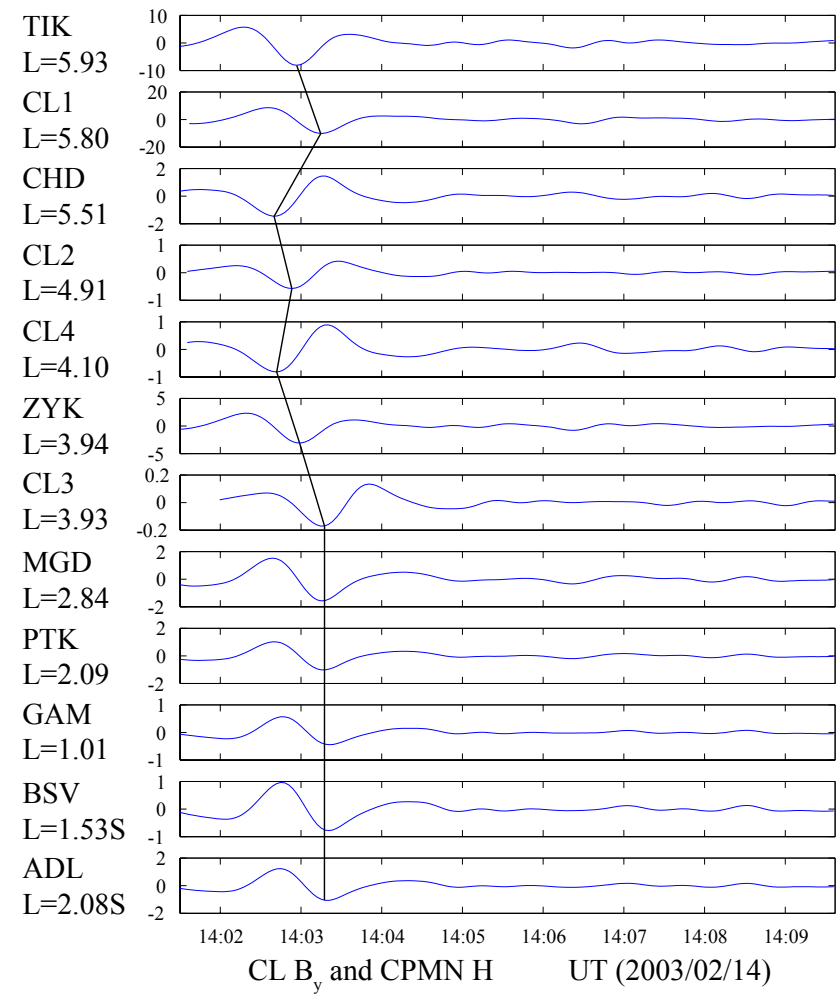

Fig. 8. The same as Fig. 7 except that the Cluster data are shifted in time so as to correct for the field-aligned propagation time from the position of each satellite to the ground. See the text for more details.

the ground. Thus obtained travel times are $6.9 \mathrm{~s}, 5.4 \mathrm{~s}, 29.5 \mathrm{~s}$, and $5.4 \mathrm{~s}$ for CL1, CL2, CL3, and CL4; these times are expected to be the time lag from the in-situ observation time at Cluster to the expected observation time at the ground footprint. If we apply this time shift to the Cluster data in Fig. 7, the result is that shown in Fig. 8. The time shift is small for CL1, CL2, and CL4, because they were located within the plasmatrough where $V_{\mathrm{A}}$ is generally high; on the other hand the time shift is pretty large (about $30 \mathrm{~s}$ ) for CL3 because CL3 was located within the plasmasphere where $V_{\mathrm{A}}$ is lower.

As a result of the time shift, the latitude dependence of the Pi 2 phase in Fig. 8 is more systematic than in Fig. 7; first let us recall that, as shown in Fig. 2, CL3 was within the plasmasphere while CL4 was outside the plasmapause. Then, Fig. 8 shows that the phase was almost constant within the plasmasphere, and that the phase was earlier just outside the plasmapause (CL4, CL2, and CHD). At larger $L$ (CL1 and TIK) the phase was later. These features will be further discussed in the next section.

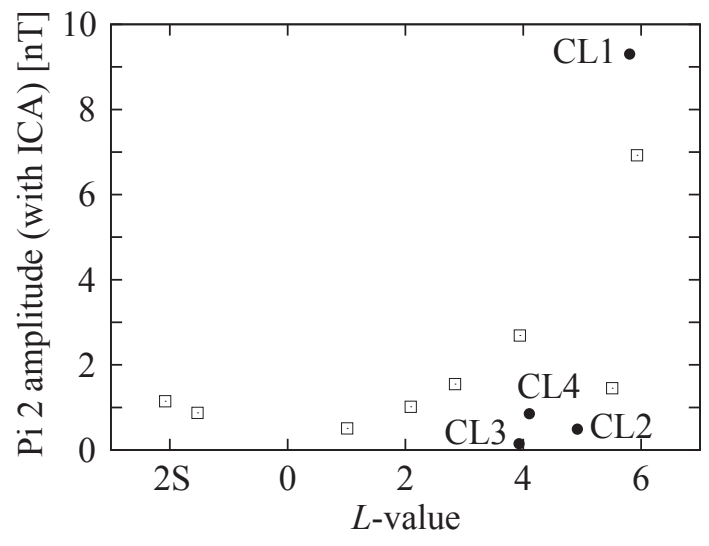

Fig. 9. Visualizes the wave amplitudes shown in Figs. 7 and 8. The horizontal axis is the $L$-value and the vertical axis is the half of the maximum peak-to-peak amplitude shown in Figs. 7 and 8. Filled circles (labelled) correspond to Cluster, while the open squares (not labelled) correspond to the CPMN ground stations.

\section{Discussion and summary}

For the Pi 2 event of this paper, only the $B_{\mathrm{y}}$ (eastward) component showed perturbations coherent with the ground $H$-component perturbations at all the four Cluster satellites; because the purpose of this paper is to study the relations between simultaneous observations by Cluster multisatellites and multiple ground magnetometers, in this paper we have concentrated on the $B_{\mathrm{y}}$ component.

One may think that the $B_{\mathrm{x}}$ (radial) component in space should have a good correlation with the ground $H$-component for cavity mode-type perturbations; it is a topic of future research to identify the reason why it was not such for our event, but we note that Collier et al. (2006) reported another case of Pi 2 observed by Cluster, in which CL2 and CL4 within the plasmasphere observed significant oscillations in $B_{\mathrm{y}}$ (see their Fig. 4); Collier et al. (2006) attributed this feature to coupling between the cavity mode and the toroidal mode, which could take place if the cavity mode had a non-zero azimuthal wave number. Perhaps our event also had a non-zero azimuthal wave number, and the big portion of the cavity mode wave energy was put into the oscillations in the azimuthal direction.

Figure 9 visualizes the wave amplitudes shown in Figs. 7 and 8. The horizontal axis is the $L$-value and the vertical axis is the half of the maximum peak-to-peak amplitude shown in Figs. 7 and 8. Filled circles (labelled) correspond to Cluster, while the open squares (not labelled) correspond to the CPMN ground stations. Figure 9 shows that the ground magnetic field amplitude of Pi 2 increases with increasing latitude at high latitudes. This tendency is consistent with that reported in past reports (see, e.g. Fig. 4 of Yumoto and the CPMN Group, 2001); the large amplitude at high latitudes could be understood in terms of the Pi 2 wave source mapped 
to the auroral latitudes. On the other hand, if we look at Cluster, the Pi 2 amplitude at CL1 was about 10 times larger than that at ground low latitudes (i.e. $\sim 1 \mathrm{nT}$ at GAM and BSV), the Pi 2 amplitudes at CL2 and CL4 were comparable to that at the ground low latitudes, and the Pi 2 amplitude at CL3 was about $1 / 10$ of that at the ground low latitudes.

If we try to sort out these amplitudes at Cluster in terms of the above-stated latitude dependence of the amplitude, CL1 is consistent because the amplitude at CL1 $(L=5.80)$ was similar to that at the adjacent (in terms of the $L$ value) higherlatitude station TIK $(L=5.93)$. On the other hand, the amplitudes at CL2, CL4 and CL3 (especially CL3) were smaller than those at the adjacent (in $L$ ) ground stations in the figure (i.e. CHD $(L=5.51)$ and ZYK $(L=3.94))$, not apparently consistent with the above-stated latitude dependence. For CL4 and CL3, we think the small amplitudes were caused because CL4 and CL3 were located at low latitudes in space $\left(16.90^{\circ}\right.$ and $-2.76^{\circ}$ in latitude; see Table 1). That is, if the observed Pi 2 was caused by fundamental mode-type oscillations of the field lines, then $B_{\mathrm{y}}$ in space is expected to have been small near the equator which corresponded to the antinode of the field-line oscillations.

On the other hand, the small amplitude at CL2 $(L=4.91)$ about half of that at nearby ground station CHD $(L=5.51)$, is rather difficult to explain, because CL2 was located at $24.57^{\circ}$ in latitude in space (see Table 1), i.e. at pretty much high latitude. We note that CL2 shows the data for which the waveforms in Figs. 6 and 7 are the most different (that is, the wave amplitude in Fig. 6 is at maximum in the latter half of the time interval of the figure), and that the maximum amplitude at CL2 in Fig. 6 is comparable to that at CHD, which also shows a fairly large difference in the waveform between Figs. 6 and 7 so that its maximum amplitude in Fig. 7 is less than half of that in Fig. 6. Our speculation here is that the area (in space) near CL2 and CHD $(L \sim 5)$ was associated with active wave-mode conversion from Pi 2 to some other type, so that the wave energy of Pi 2 was lost to some extent at CL2 and CHD.

We saw above that, by taking into account the field-aligned Alfven-wave travel time from Cluster to the ground (Fig. 8), the latitude-phase relation of Pi 2 becomes more systematic than otherwise (Fig. 7). From this we think that the data corresponding to the plasmatrough (CL4 $(L=4.10)$ and larger $L$ ) were caused by the earthward propagation of the Pi 2 signal. We also note that Uozumi et al. (2009) explained their observations of Pi 2's at TIK and CHD in terms of the Pi 2 wave propagation.

Past studies show that the source region of Pi 2 is usually field-aligned mapped to the ground more poleward than TIK $(L=5.93)$. Then, at first glance, the delay in the phase at CL1 and TIK from CHD-CL4 may appear to contradict with the interpretation in terms of the $\mathrm{Pi} 2$ propagation; however, in fact it does not, as follows.

Figure 1a of Uozumi et al. (2007) and Fig. 2a of Chi et al. (2009) illustrate how the Pi 2 signal propagates from the source region to the ground: for the $L$ of CL1 and CHD, the travel path is such that the Pi 2 signal first propagates in the equatorial plasmasheet toward the Earth as the fast-mode wave, and when the signal reaches the field line running through the ground station, it is mode-converted from the fast mode to the Alfven mode and propagates along the field line toward the Earth. Figure 2b of Chi et al. (2009) illustrates that the Alfvenic field-aligned travel time decreases sharply with decreasing $L$; it is so because $V_{\mathrm{A}}$ sharply increases with decreasing $L$ in the trough (see, e.g. Fig. 1, top panel of Fujita et al., 2001 for illustration). This is the reason why the Pi 2 signal in the mid-latitude trough reaches the ground faster than the Pi 2 signal in the higher-latitude trough. (It is to be noted that the majority of the Alfvenic field-aligned travel time is spent near the equatorial plasmasheet, because $V_{\mathrm{A}}$ increases very quickly as the Alfven wave field-aligned propagates from the equator toward higher latitudes in space; see, e.g. Fig. 1, bottom panel of Fujita et al. (2001) for illustration. This is the reason why the estimated field-aligned travel time is not significant for CL1, CL2, and CL4 in Fig. 8; these satellites were located off the equator.)

In Fig. 8, the phase delay is less for TIK than for CL1, although a natural expectation is the opposite (as explained above). We think this happened due to azimuthal propagation of the Pi 2 signal with TIK located significantly westward of the other observation points (see Table 1). That is, at the event time, TIK was located at 22.6 LT while the other ground stations were located at 23.3-24.6 LT. If the Pi 2 source region of our event was near 22.5 MLT which is the statistical average for Pi 2 observed by CPMN (Uozumi et al., 2004), it is expected that the eastward propagation of the Pi 2 signal from the source region made TIK observe the Pi 2 faster than the other stations. Similar azimuthal propagation effect was reported by Uozumi et al. (2004).

In Fig. 8, the monotonic delay in phase from CL4 to CL3 is likely to have been caused by the $\mathrm{Pi} 2$ propagation in the plasmapause boundary layer where $V_{\mathrm{A}}$ sharply decreases with decreasing $L$ (see the region near $L=5$ in Fig. 1 of Lee and Lysak, 1999 or in Fig. 1 (top) of Fujita et al., 2001 for illustration). The plasmasphere, including CL3 and the lower-latitude ground stations (MGD-ADL), was hit by this Pi 2 signal, and the cavity mode would have formed in the plasmasphere, as reported in many past papers. As expected at the beginning of this work, we could confirm this propagation and hitting process by the combination of the Cluster multisatellites in space and the CPMN ground magnetometer network, and with the aid of ICA to separate out other waves with spectral content similar to Pi 2.

Whether the signals observed at MGD-ADL (Fig. 8) correspond to the cavity-mode oscillation of the plasmasphere in response to the above-presented hitting process, or to the propagation process itself of the Pi 2 signal within the plasmasphere, leaves room for argument, since we have no other satellites within the plasmasphere; ground observation only is not necessarily sufficient to separate out field-aligned 
travel time from radial travel time of Pi 2. For example, Uozumi et al. (2000) statistically showed that Pi 2 signals propagating within the plasmasphere were observed earlier at smaller $L$ on the ground (see their Fig. 3), unlike the one shown in this paper, and successfully explained it by their model cited above. On the other hand for the event of this paper, the phase is very coherent within the plasmasphere, but it might also be explained with a propagation model with different model parameters.

As a multisatellite observation within the plasmasphere, Collier et al. (2006) presented a case in which CL1, CL2, and CL4 were located within the plasmasphere, and they found evidence for the cavity mode. Since their Pi 2 oscillated about three times in its wave packet while our Pi 2 (its common part identified by ICA) oscillated only about once (Fig. 6-8), there may be an argument such a short wave is not enough to establish the standing oscillation of the cavity; however, we note Fujita et al. (2002) simulated a Pi 2 by inputting a single pulse in their simulation code, and they showed that the entire plasmasphere started to oscillate in the cavity mode very fast. Thus, the number of oscillations of Pi 2 may not be relevant for the establishment of a cavity wave. A reasonable extension of this earlier study by Collier et al. (2006) would be to analyze cases in which a Pi 2 is simultaneously monitored in the plasmasphere by a ground magnetometer chain and multisatellites along the same meridian, to distinguish the field-aligned travel time from the radial travel time with observations; it is a topic of future research.

Acknowledgements. Work at JHU/APL was supported by NASA grants NNX07AG07G and NNX09AF46G. The FastICA package is free software under copyright 1996-2005 by Hugo Gävert, Jarmo Hurri, Jaakko Särelä, and Aapo Hyvärinen.

Topical Editor I. Daglis thanks A. Collier and another anonymous referee for their help in evaluating this paper.

\section{References}

Baker, K. B. and Wing, S.: A new magnetic coordinate system for conjugate studies at high latitudes, J. Geophys. Res., 94(A7), 9139-9144, 1989.

Balogh, A., Carr, C. M., Acuña, M. H., Dunlop, M. W., Beek, T. J., Brown, P., Fornacon, K.-H., Georgescu, E., Glassmeier, K.H., Harris, J., Musmann, G., Oddy, T., and Schwingenschuh, K.: The Cluster Magnetic Field Investigation: overview of in-flight performance and initial results, Ann. Geophys., 19, 1207-1217, doi:10.5194/angeo-19-1207-2001, 2001.

Carpenter, D. L. and Anderson, R. R.:, An ISEE/Whistler model of equatorial electron density in the magnetosphere, J. Geophys. Res., 97, 1097-1108, 1992.

Chi, P. J., Russell, C. T., and Ohtani, S.: Substorm onset timing via traveltime magnetoseismology, Geophys. Res. Lett., 36, L08107, doi:10.1029/2008GL036574, 2009.

Collier, A. B., Hughes, A. R. W., Blomberg, L. G., and Sutcliffe, P. R.: Evidence of standing waves during a $\mathrm{Pi} 2$ pulsa- tion event observed on Cluster, Ann. Geophys., 24, 2719-2733, doi:10.5194/angeo-24-2719-2006, 2006.

Escoubet, C. P., Fehringer, M., and Goldstein, M.: Introduction The Cluster mission, Ann. Geophys., 19, 1197-1200, doi:10.5194/angeo-19-1197-2001, 2001.

Fujita, S., Mizuta, T., Itonaga, M., Yoshikawa, A., and Nakata, H.: Propagation property of transient MHD impulses in the magnetosphere-ionosphere System: The 2D model of the Pi2 pulsation, Geophys. Res. Lett., 28(11), 2161-2164, 2001.

Fujita, S., Nakata, H., Itonaga, M., Yoshikawa, A., and Mizuta, T.: A numerical simulation of the $\mathrm{Pi} 2$ pulsations associated with the substorm current wedge, J. Geophys. Res., 107, A3, doi:10.1029/2001JA900137, 2002.

Hsu, T.-S. and McPherron, R. L.: A statistical study of the relation of Pi 2 and plasma flows in the tail, J. Geophys. Res., 112, A05209, doi:10.1029/2006JA011782, 2007.

Hyvärinen, A.: Fast and robust fixed-point algorithms for Independent Component Analysis, IEEE T. Neural Network., 10(3), 626-634, 1999.

Hyvärinen, A., Karhunen, J., and Erkki, O.: Independent Component Analysis, John Wiley \& Sons, Inc., ISBN 9780471405405 , doi:10.1002/0471221317, 2001.

Gustafsson, G., Boström, R., Holback, B., Holmgren, G., Lundgren, A., Stasiewicz, K., Åhlén, L., Mozer, F. S., Pankow, D., Harvey, P., Berg, P., Ulrich, R., Pedersen, A., Schmidt, R., Butler, A., Fransen, A. W. C., Klinge, D., Thomsen, M., Fälthammar, C.-G., Lindqvist, P.-A., Christenson, S., Holtet, J., Lybekk, B., Sten, T. A., Tanskanen, P., Lappalainen, K., and Wygant, J.: The Electric Field and Wave Experiment for Cluster, Space Sci. Rev., 79, 137-156, 1997.

Lee, D.-H. and Lysak, R. L.: MHD waves in a three-dimensional dipolar magnetic field: A search for Pi 2 pulsations, J. Geophys. Res., 104, 28691-28699, 1999.

Olson, J. V.: Pi2 pulsations and substorm onsets: A review, J. Geophys. Res., 104, 17499-17520, 1999.

Pedersen, A., Lybekk, B., André, M., Eriksson, A., Masson, A., Mozer, F. S., Lindqvist, P.-A., Décréau, P. M. E., Dandouras, I., Sauvaud, J.-A., Fazakerley, A., Taylor, M., Paschmann, G., Svenes, K. R., Torkar, K., and Whipple, E.: Electron density estimations derived from spacecraft potential measurements on Cluster in tenuous plasma regions, J. Geophys. Res., 113, A07S33, doi:10.1029/2007JA012636, 2008.

Rème, H., Aoustin, C., Bosqued, J. M., Dandouras, I., Lavraud, B., Sauvaud, J. A., Barthe, A., Bouyssou, J., Camus, Th., CoeurJoly, O., Cros, A., Cuvilo, J., Ducay, F., Garbarowitz, Y., Medale, J. L., Penou, E., Perrier, H., Romefort, D., Rouzaud, J., Vallat, C., Alcaydé, D., Jacquey, C., Mazelle, C., d'Uston, C., Möbius, E., Kistler, L. M., Crocker, K., Granoff, M., Mouikis, C., Popecki, M., Vosbury, M., Klecker, B., Hovestadt, D., Kucharek, H., Kuenneth, E., Paschmann, G., Scholer, M., Sckopke, N., Seidenschwang, E., Carlson, C. W., Curtis, D. W., Ingraham, C., Lin, R. P., McFadden, J. P., Parks, G. K., Phan, T., Formisano, V., Amata, E., Bavassano-Cattaneo, M. B., Baldetti, P., Bruno, R., Chionchio, G., Di Lellis, A., Marcucci, M. F., Pallocchia, G., Korth, A., Daly, P. W., Graeve, B., Rosenbauer, H., Vasyliunas, V., McCarthy, M., Wilber, M., Eliasson, L., Lundin, R., Olsen, S., Shelley, E. G., Fuselier, S., Ghielmetti, A. G., Lennartsson, W., Escoubet, C. P., Balsiger, H., Friedel, R., Cao, J.-B., Kovrazhkin, R. A., Papamastorakis, I., Pellat, R., Scudder, J., and Sonnerup, B.: 
First multispacecraft ion measurements in and near the Earth's magnetosphere with the identical Cluster ion spectrometry (CIS) experiment, Ann. Geophys., 19, 1303-1354, doi:10.5194/angeo19-1303-2001, 2001.

Sutcliffe, P. R. and Yumoto, K.: On the cavity mode nature of low latitude Pi 2 pulsations, J. Geophys. Res., 96, 1543-1551, 1991.

Takahashi, K. and Anderson, B. J.: Distribution of ULF energy $(f<80 \mathrm{mHz})$ in the inner magnetosphere: A statistical analysis of AMPTEE CCE magnetic field data, J. Geophys. Res. 97, 10751-10773, 1992.

Takahashi, K., Ohtani, S., and Anderson, B. J.: Statistical analysis of Pi 2 pulsations observed by the AMPTE CCE spacecraft in the inner magnetosphere, J. Geophys. Res., 100, 21929-21941, 1995.

Takahashi, K., Ohtani, S., Hughes, W. J., and Anderson, R. R.: CRRES observation of Pi2 pulsations: Wave mode inside and outside the plasmasphere, J. Geophys. Res., 106, 15567-15581, 2001.

Takahashi, K., Lee, D., Nose, M., Anderson, R. R., and Hughes, W. J.: CRRES electric field study of the radial mode structure of Pi 2 pulsations, J. Geophys. Res., 108(A5), 1210, doi:10.1029/2002JA009761, 2003.

Tokunaga, T., Kohta, H., Yoshikawa, A., Uozumi, T., and Yumoto, K.: Global features of Pi 2 pulsations obtained by independent component analysis, Geophys. Res. Lett., 34, L14106, doi:10.1029/2007GL030174, 2007.

Torkar, K., Riedler, W., Escoubet, C. P., Fehringer, M., Schmidt, R., Grard, R. J. L., Arends, H., Rüdenauer, F., Steiger, W., Narheim, B. T., Svenes, K., Torbert, R., André, M., Fazakerley, A., Goldstein, R., Olsen, R. C., Pedersen, A., Whipple, E., and Zhao, H.: Active spacecraft potential control for Cluster implementation and first results, Ann. Geophys., 19, 1289-1302, doi:10.5194/angeo-19-1289-2001, 2001.

Tsyganenko, N. A.: Effects of the solar wind conditions on the global magnetospheric configuration as deduced from data-based field models, Proc. Third International Conference on Substorms (ICS-3), Versailles, France, 12-17 May 1996, ESA SP-389, October 1996.
Uozumi, T., Yumoto, K., Kawano, H., Yoshikawa, A., Olson, J. V., Solovyev, S. I., and Vershinin, E. F.: Characteristics of energy transfer of Pi 2 magnetic pulsations: Latitudinal dependence, Geophys. Res. Lett., 27(11), 1619-1622, 2000.

Uozumi, T., Yumoto, K., Kawano, H., Yoshikawa, A., Ohtani, S., Olson, J. V., Akasofu, S.-I., Solovyev, S. I., Vershinin, E. F., Liou, K., and Meng, C.-I.: Propagation characteristics of Pi 2 magnetic pulsations observed at ground high latitudes, J. Geophys. Res., 109, A08203, doi:10.1029/2003JA009898, 2004.

Uozumi, T., Kawano, H., Yoshikawa, A., Itonaga, M., and Yumoto, K.: Pi 2 source region in the magnetosphere deduced from CPMN data, Planet. Space Sci., 55, 849-857, 2007.

Uozumi, T., Abe, S., Kitamura, K., Tokunaga, T., Yoshikawa, A., Kawano, H., Marshall, R., Morris, R. J., Shevtsov, B. M., Solovyev, S. I., McNamara, D. J., Liou, K., Ohtani, S., Itonaga, M., and Yumoto, K.: Propagation characteristics of Pi 2 pulsations observed at high- and low-latitude MAGDAS/CPMN stations: A statistical study, J. Geophys. Res., 114, A11207, doi:10.1029/2009JA014163, 2009.

Yeoman, T. K. and Orr, D.: Phase and spectral power of midlatitude Pi2 pulsations: Evidence for a plasmaspheric cavity resonance, Planet. Space Sci., 37, 1367-1383, doi:10.1016/00320633(89)90107-4, 1989.

Yumoto, K. and the 210MM Magnetic Observation Group: The STEP 210 magnetic meridian network project, J. Geomagn. Geoelectr., 48, 1297-1310, 1996.

Yumoto, K. and the CPMN Group: Characteristics of Pi 2 magnetic pulsations observed at the CPMN stations: A review of the STEP results, Earth Planets Space, 53, 981-992, 2001. 